\title{
La mémoire du Moyen Âge et Thomas Owen
}

\section{The Memory of the Middle Ages and Thomas Owen}

\author{
Bernard Ribémont \\ Université d'Orléans \\ e-mail: bernard.ribemont@univ-orleans.fr
}

\begin{abstract}
Thomas Owen is well-known as a master of the fantastic $20^{\text {th }}$ century's short stories. This genre is often influenced by the medieval fiction, in its atmosphere, 'colour' and moreover throught precise figures, situations and, sometimes, well specific works, specially arthurian. The case of Owen offers the particular interest of not really a rewriting or even a genuine reception but, supported by a knowledge of some texts, of a literary construction founded on the author's appreciation of a 'spirit of the Middle Ages'.
\end{abstract}

Keywords: Memory, Fantasy, Medievalization, Medieval Culture, « Troubadour », Courtly Love

La littérature européenne, depuis la Renaissance, est, considérée dans son ensemble, profondément marquée par la culture médiévale. Que ce soit en se situant dans un système d'opposition, d'ailleurs souvent purement rhétorique et, je dirais, 'publicitaire', comme chez bien des Humanistes ou, au contraire, en utilisant explicitement des thèmes et motifs, des situations diégétiques ou des événements historiques, beaucoup d'auteurs, romanciers et poètes, ont été influencés par un Moyen Âge que le lecteur est parfois surpris de rencontrer en des lieux où il ne l'attend pas le moins du monde a priori, comme par exemple - pour citer un cas particulièrement étonnant - chez Jean Paulhan qui a lu et utilisé un auteur du XIII ${ }^{\mathrm{e}}$ siècle fort loin de faire partie des lectures familières d'un lettré français du $X X^{\mathrm{e}}$ siècle, à savoir Brunetto Latini ${ }^{1}$. L'on pourra donc parler d'une forme de permanence de la culture

\footnotetext{
${ }^{1}$ Brunetto Latini, notaire florentin, est l'auteur, entre autres, d'une encyclopédie en français $\left(\mathrm{XIII}^{\mathrm{e}} \mathrm{s}\right.$.) qui connut un fort succès au Moyen Âge (Le Livre du trésor) et qui ne fait pourtant pas partie
} 
et, plus particulièrement, des Lettres médiévales, depuis les premiers imprimés diffusant romans, chroniques et chansons de geste jusqu'à des réécritures contemporaines, telles par exemple l'Erec et Enide de Montalban, en passant par la littérature populaire de la Bibliothèque bleue - dont la diffusion s'étend à la Belgique ${ }^{2}-$, ou encore les massifs genres gothique et « troubadouresque » du XIX ${ }^{\mathrm{e}}$ siècle.

L'étude de la mémoire du Moyen Âge ou, pour le dire différemment, de la réception des œuvres médiévales dans les littératures postérieures, posent un certain nombre de problèmes, dont certains relatifs aux sources. Sans tomber en effet dans un positivisme trop marqué relevant d'une systématique Quellenforschung, il est toujours intéressant de s'interroger sur les éventuels hypotextes dont tel ou tel auteur a eu connaissance. De fait, cette interrogation sur les sources a surtout pour intérêt d'avancer dans l'analyse de ce que j'appellerai la fabrication d'une littérature médiévalisante. Les difficultés inhérentes à une recherche de source, le plus souvent vaine, sauf si l'auteur moderne est suffisamment explicite à ce sujet, ouvrent la voie à une analyse des procédés littéraires mis en œuvre qui, en outre, mettent en lumière ce qu'un auteur a reçu, compris, déformé d'une culture qui le plus souvent lui échappe et l'influence sous forme de clichés.

La façon dont un texte, ouvrage entier ou simples pages insérées, propose un discours médiévalisant relève de plusieurs démarches, que l'on peut considérer selon trois cas principaux. Le premier relève d'une récriture qui s'apparente à une adaptation d'une source qui est soit clairement revendiquée, soit qui apparait de façon suffisamment explicite au lecteur, du moins au lecteur averti; il s'agit le plus généralement de textes chevaleresques ou épiques appartenant au corpus le plus connu d'un lettré ou d'une personne cultivée : œuvres de Chrétien de Troyes ou de ses continuateurs, Chanson de Roland, Renaut de Montauban, etc. J'évoquais précédemment l'Erec et Enide de Montalban, inspiré du premier roman de Chrétien ; on pourrait citer la seule pièce de théâtre de Julien Gracq, Le Roi pêcheur jouée en $1940^{3}$, qui prend sa source dans le Parzifaal de Wolfram von Eschenbach (avec toutefois un passage par Wagner ${ }^{4}$ ) ou, plus récemment et dans le domaine de la bande dessinée, le Bel inconnu de Nathalie Ferlut, relecture du roman de Renaut de Beaujeu, composé au XIII ${ }^{\mathrm{e}}$ siècle. De façon encore plus emblématique et systéma-

\footnotetext{
des auteurs les plus connus d'un public non spécialiste ; on lui doit également une Rettorica très influencée par Cicéron, dont le notaire traduit des passages entiers en italien. Voir à ce sujet Chagas Oliveira E. (2008). «A Descoberta da Nova Retórica: Jean Paulhan e Brunetto Latini. La Découverte de la Nouvelle Rhétorique : Jean Paulhan et Brunetto Latini ». Ideação, (Núcleo Interdisciplinar de Estudos e Pesquisas em Filosofia), 18/12, 2008, 17-32.

${ }^{2}$ Voir à ce propos Ruelens Ch. (1872). «La Bibliothèque bleue en Belgique », Le Bibliophile belge, 7, 59-69 et 200-204.

${ }^{3}$ On pourra noter un certain goût de Gracq pour le Moyen Âge, en se référant au Château d'Argol ou au Beau ténébreux. Gracq indique d'ailleurs dans l'avant-propos de sa pièce que ce qu'il nomme les mythes du Moyen Âge sont des « œuvres ouvertes ».

${ }^{4}$ Voir Marot P. (1995). "Le Roi pêcheur : Le Graal ou l'envers de la représentation », Cahiers de l'Association internationale des études françaises, 47, 115-134.
} 
tique se présente la récriture que Michel Rio a faite du cycle du Lancelot-Graal avec sa trilogie Merlin, Morgane, Arthur ${ }^{5}$. Une autre catégorie, à vrai dire assez proche de la première, réunit des textes qui s'affichent comme médiévalisants et qui s'alimentent à diverses sources, le plus souvent non identifiables avec précision, mais qui reprennent et adaptent des motifs et des scènes topiques, comme le fait par exemple Cocteau en 1937 dans ses Chevaliers de la Table ronde, œuvre à la lecture de laquelle apparaît manifestement la connaissance du Lancelot en prose ${ }^{6}$. La troisième catégorie, sans doute la plus foisonnante, concerne des textes qui revêtent une tonalité médiévalisante, résultante de diverses composantes - personnages, décors, clins d'oeil référentiels, etc. - tonalité qui vise à placer le lecteur dans une certaine ambiance, en lui soumettant éventuellement certaines interrogations, certaines leçons dont les auteurs peuvent penser que la référence médiévale les souligne, les met en lumière ; on pourra penser à l'exemple d'Il cavaliere inesistente de Calvino ou encore à la foison de ces polars médiévaux qui, depuis les créations l'Ellis Peeters, connaissent un succès international. Les catégories mentionnées ici n'ont évidemment pas de frontière étanche et bien des œuvres relèvent des trois à la fois, tout particulièrement un certain nombre de textes appartenant au genre de la littérature fantastique.

Thomas Owen, on le sait, est connu du public comme un maître de la nouvelle fantastique. La seule évocation de la littérature fantastique nous renvoie vers la fin $\mathrm{du} \mathrm{XVIII}^{\mathrm{e}}$ et surtout vers le $\mathrm{XIX}^{\mathrm{e}}$ siècle et donc vers un imaginaire qui s'actualise très souvent dans un décor médiéval ou pseudo-médiéval, comme on peut le voir chez Walpole ou Radcliffe, chez Hoffmann et Nodier.

Considérons donc, du point de vue d'une écriture médiévalisante, Owen comme un écrivain cultivant la nouvelle et ce, au $X^{\mathrm{e}}$ siècle. Cette élémentaire caractérisation appelle quelques remarques. À l'époque où Owen compose ses nouvelles, le genre a acquis ses lettres de noblesse et surtout un certain nombre de principes élaborés en particulier chez Poe et James. Inutile ici de reprendre les analyses fort connues de Todorov; je n'insisterai que sur le caractère énigmatique, provocant l'hésitation, qui caractérise le fantastique en le distinguant du merveilleux. Or, le Moyen Âge, comme la vaste thèse de Francis Dubost l'a bien montré ${ }^{7}$, joue sans le savoir sur les frontières de ces catégories modernes, du moins dans le jeu des

\footnotetext{
${ }^{5}$ Pomel F. (2005). « Michel Rio et le modèle textuel médiéval : filiation et paternité littéraires », in Images du Moyen Âge. Rennes : Presses Universitaires de Rennes ; Pomel F. (2010). « Michel Rio : les réécritures arthuriennes », Cahiers de recherches médiévales et humanistes, 19, 145-149; Ribémont B. (2006). "Michel Rio: une réécriture de la légende arthurienne ou la mystique de la loi», in S. Wodianka \& D. Rieger (Eds.), Mythosaktualisierungen. Tradierungs-und Generierungspotentiale einer alten Errinerugsform (pp. 193-209), Berlin - New York : De Gruyter.

${ }^{6}$ Schneider M. (1985). «Cocteau et le Moyen Âge », Cahiers Jean Cocteau, 10, 259-265 ; Baudry R. (1996). «Le Lancelot-Guenièvre de Jean Cocteau ou les Avatars du Mythe Amours, Sosies et Substitutions ». CRMH, 37-49.

${ }^{7}$ Dubost F. (1991). Aspects fantastiques de la littérature narrative médiévale (XII ${ }^{e}$-XIII ${ }^{e}$ s.). L'autre, l'ailleurs, l'autrefois, 2 vol., Paris : Champion.
} 
procédés littéraires, comme on le voit par exemple dans le Roman de Mélusine de Jean d'Arras. Par ailleurs, le $\mathrm{XX}^{\mathrm{e}}$ siècle a, beaucoup plus qu'on ne pourrait le penser, plongé de nombreuses racines dans le Moyen Âge et l'idée qu'il pouvait s'en faire. Notre contemporanéité en offre d'ailleurs un aboutissement spectaculaire et révélateur du cheminement opéré auparavant, avec un Moyen Âge omniprésent au cinéma, dans la BD, dans la publicité, les séries télévisées, les jeux vidéo, etc.

Cet engouement pour le médiéval repose sur plusieurs éléments. Tout d'abord un héritage. Si l'héritage passant par le fantastique et le romantisme est une évidence, on n'oubliera pas que la fin du $\mathrm{XIX}^{\mathrm{e}}$ a aussi produit des effets majeurs dans ce domaine à travers plusieurs facteurs : 1) dans un contexte purement littéraire, il faut noter les rapports, au demeurant complexes, entre les courants dandy, décadent, symboliste et le Moyen Âge : un Huysmans défenseur de l'art médiéval, un Rémi de Gourmont vantant les mérites du latin médiéval par exemple ; 2) le développement des études folkloristes et corrélativement, celui des recherches sur les 'racines populaires' des cultures conduisant à la diffusion et à la réexploitation de bien des légendes médiévales et l'on pensera en tout premier lieu à Richard Wagner ; 3) l'émergence des études médiévales universitaires avec la création de chaires d'histoire littéraire du Moyen Âge ; corrélativement, le souci des premiers médiévistes de diffuser ce qu'ils considéraient comme les chefs d'œuvre de la littérature médiévale, en offrant des traductions et des manuels ; que l'on songe ici à la Chanson de Roland ou au Tristan et Iseut de Joseph Bédier. On ajoutera le succès traversant les siècles d'œuvres foisonnantes et donc susceptibles de multiples interprétations et incompréhension: La Divina commedia, le Decameron, les Canterbury Tales, Le Morte D'Arthur de Thomas Mallory, etc.

Le $\mathrm{XX}^{\mathrm{e}}$ siècle européen dispose donc, si j'ose dire, d'un arsenal à la fois vaste et hétéroclite concernant l'époque médiévale ${ }^{8}$. Quelles qu'en soient les composantes, même les plus savantes, cet ensemble est marqué en profondeur par deux tendances : l'une est issue d'un $\mathrm{XVI}^{\mathrm{e}}$ siècle largement relayé par les Lumières qui fait du Moyen Âge une période d'obscurantisme et de superstitions. L'autre, venue directement du Romantisme - et l'État belge est établi en plein Romantisme ! -, offre l'image d'un Moyen Âge à la fois noir et lumineux. Sur cet écran quelque peu contrasté, les différents travaux de réhabilitation de la culture médiévale projetteront le possible d'un Moyen Âge renversé, d'un Moyen Âge 'rose' dont les interprétations outrées et emplies de contresens de l'amour courtois sont un vecteur particulièrement fort. Pour résumer, le Moyen Âge se creuse de plus en plus au $\mathrm{XX}^{\mathrm{e}}$ siècle comme un des espaces privilégiés de l'expression de tous les fantasmes, y compris sexuels ${ }^{9}$, des auteurs usant de médiévalisation.

\footnotetext{
${ }^{8}$ Michel Rio, dans l'introduction des ses romans 'arthuriens' parle de « bric-à-brac ».

${ }^{9}$ On le voit en particulier dans la bande dessinée pour adulte (par ex. Le Ruistre de Jean-Charles Kraehn).
} 
Il est donc normal en quelque sorte que le genre de la nouvelle fantastique trouve à s'alimenter à ce réservoir de motifs et d'images qui s'est cristallisé au fil des siècles. Il convient me semble-t-il de noter également, à propos de Thomas Owen, que la littérature belge hérite d'une tradition médiévale particulièrement développée qui prend son origine dans le dynamisme culturel de la cour de Bourgogne à la fin du Moyen Âge. Je rappellerai ici pour mémoire que les imprimeurs reproduisirent, dès avant 1550 , de nombreux textes, tous genres confondus ${ }^{10}$, fort goûtés de l'aristocratie bourguignonne, textes qui souvent avaient bénéficié de luxueuses copies manuscrites. On pensera également au mécénat de grands bourgeois attachés aux riches villes du Nord, Bruges, Gand, Ypres. Il convient peut-être de rappeler que le roman fondateur de la culture belge de langue française Thyl Ulenspiegel de Charles De Coster, publié en 1867 avec une illustration de Félicien Rops, se déroule à l'époque de Charles V. On pensera aussi à Maeterlinck traducteur de Ruysbroeck. Et les ducs de Bourgogne de fasciner encore des écrivains contemporains tel Gaston Compère avec son Je soussigné Charles le Téméraire duc de Bourgogne ou René Kalisky avec son Charles le Téméraire ou l'autopsie d'un prince ${ }^{11}$.

Or Owen est de toute évidence un écrivain de culture et, si son succès est important, voire 'populaire', il est bien des aspects de ses nouvelles qui sont d'un abord loin de l'immédiateté ; en particulier, Owen se plaît à incorporer de très nombreuses références, de multiples clins d'œil clairement destinés à produire la reconnaissance d'un certain public : la liste des écrivains, metteurs en scène, compositeurs cités par Owen serait ainsi longue à établir. Je remarquerai au passage que notre auteur a de toute évidence une certaine connaissance érudite des textes médiévaux (on ne peut le soupçonner, à l'époque où il écrit, d'avoir utilisé internet pour avoir seulement quelques titres à mentionner et faire ainsi le cuistre !). Dans Portrait d'un inconnu, histoire d'un professeur Sardon au destin tragique se terminant sur l'évocation de la célébrissime licorne, Owen fait preuve de la connaissance d'auteurs de Bestiaires qui, ici encore, ne peuvent être soupçonnés de faire partie des lectures ordinaires d'un écrivain du XX $\mathrm{X}^{\mathrm{e}}$ siècle : Richard de Fournival, Guillaume le Clerc de Normandie et, plus confidentiel encore, Philippe de Thaon, dont l'œuvre écrite en anglonormand et non traduite était peu accessible. Dans ses essais, Owen montre aussi une certaine connaissance, à travers la lecture de Maritain, de Thomas d'Aquin ${ }^{12}$.

\footnotetext{
${ }^{10}$ Romans en prose comme le Perceforest, mises en prose : Gérard de Nevers, Girart de Roussillon, Renaut de Montauban, Clamadès, Baudouin de Flandre... ; Cent Nouvelles nouvelles, œuvres de George Chastelain, de Jean Molinet (entre autres, son Roman de la Rose moralisé), d'Olivier de la Marche, de Molinet, etc.

${ }^{11}$ Voir Lope H. J. et Neuschäfer A. (ed.) (1998). René Kalisky (1936-1981) et la hantise de l'Histoire, éd., Frankfurt/Main - Berlin - New York - Paris - Wien : Peter Lang ; Silvestri A. (2003) « Charles le Téméraire ou l'autopsie d'un prince de René Kalisky : un défi contre la 'désexistence' », in Les écrivains francophones interprètes de l'Histoire : entre filiation et dissidence : Actes du colloque de Cerisy-la-Salle, du 2 au 9 septembre 2003, Bruxelles : Peter Lang, 435-456.

${ }^{12}$ Euvres complètes (1998), t. 4, Bruxelles : Lefrancq, 503-505.
} 
Il serait toutefois très abusif de considérer Thomas Owen comme un auteur médiévalisant, bien peu de ses textes se référant à cette période. Cependant, et c'est ce qui m'intéresse ici, cet auteur de fait résolument moderne, quelque peu fasciné par les États-Unis à propos desquels il nous livre ses impressions, n'échappe pas à la tentation médiévalisante. Comme nous allons le voir, Owen ne récrit pas, n'emprunte pas directement, ne fait que très peu de références explicites mais il procède, de façon très subtile, en créant une atmosphère qui repose, sinon sur une connaissance aiguisée des textes médiévaux, en tout cas sur une compréhension très fine de ce que certains de ces textes, comme le Conte du graal de Chrétien de Troyes, peuvent véhiculer. Les procédés médiévalisants d'Owen sont donc essentiellement allusifs, reposant sur une fine compréhension d'éléments de la littérature médiévale, éléments à l'aide desquels il va guider et dérouter son lecteur.

Un procédé médiévalisant qu'Owen aime à employer et qui est parfaitement adapté à la nouvelle fantastique est ce que j'appellerai un 'indice à décrypter'. Il s'agit en fait d'offrir au lecteur averti une référence explicite, hypothèse première qui pourra permettre à ce lecteur détective de comprendre ce qui sous-tend une histoire apparemment décalée par rapport à la référence initiale. Le Tétrastome, paru en 1988, offre un bon exemple de cette façon de Thomas Owen. La plupart des récits composant le Tétrastome repose sur une métamorphose, thématique souvent à l'œuvre dans maint conte et qui est inscrite dans les lais bretons, comme celle du loup-garou (Bisclarvet de Marie de France) qui fait d'ailleurs l'objet d'un des récits du Tétrastome. Dans la nouvelle À la fée zibeline, Owen, comme très souvent, met en scène une femme ; celle-ci se nomme Bertie-Bertie et possède un magasin portant l'enseigne « à la fée Zibeline ». Bertie-Bertie, venant de se présenter, s'adresse au narrateur-héros en lui demandant « vous ne trouvez pas que c'est bien ? », ce à quoi ce dernier répond aussitôt, en jouant sur la rime « oui, cela me fait penser à la fée Mélusine $»^{13}$. La suite du récit semble n'avoir aucun rapport avec la célèbre fée inventée par Jean d'Arras pour célébrer la maison de Lusignan. Bertie-Bertie, assez volontaire et impérieuse, s'arrange pour rejoindre le héros chez lui. Puis elle passe dans la salle de bains en lui claquant la porte au nez et en lui signifiant son interdiction de la suivre. Il retrouve peu de temps après la femme dans son lit. C'est en la caressant que sa main rencontre une queue de véritable zibeline: Bertie-Bertie s'enfuit alors du lit et son partenaire voit devant lui une femme nue, effectivement munie de ce curieux attribut anatomique. La femme disparaît alors. Le lecteur averti goûtera alors le signe de reconnaissance que lui adresse Owen qui avait donné un indice en mentionnant Mélusine. Bertie-Bertie est une nouvelle Mélusine, loin et près à la fois de la femme serpent; Owen a égrené certains éléments de citation, mais réadaptés, voire biaisés. Si Mélusine interdit à Raimondin de la voir lorsqu'elle part dans son bain, Bertie-Bertie interdit au narrateur-personnage de la voir dans la

\footnotetext{
${ }^{13}$ Euvres complètes, t. 4, $1998: 88$.
} 
salle de bain. Si Mélusine, dans son bain, se révèle une femme-serpent, Bertie-Bertie apparaît comme une femme-zibeline à la sortie de la salle de bains. Et quand Raimondin enfreint son serment et découvre la nature serpentine de sa femme, celle-ci s'enfuit, comme la femme-zibeline de la nouvelle d'Owen. Le lecteur féru de légendes médiévales pourra d'ailleurs goûter encore plus les clins d'œil que lui adresse Owen avec la nouvelle qui suit de peu, intitulée plus significativement La serpentine, titre qui, de toute évidence, fait le lien avec l'allusion précédente à Mélusine. Ici, le héros va se retrouver, rêve ou réalité - toujours l'hésitation propre au genre -, avec une femme-serpent.

La nuit au château, parue en 1975 dans le groupe Le Rat Kaviar, est une nouvelle également très révélatrice de la méthode d'Owen : la scène a pour cadre un moment d'une guerre indéterminée, à une époque où la cavalerie est une pièce de la stratégie militaire. Le narrateur est un capitaine qui arrive dans un château dans la pièce principale duquel il pénètre à cheval. L'action est somme toute très banale et relève de topoï de la littérature fantastique, topoï qu'Owen n'hésite d'ailleurs pas à utiliser de façon récurrente dans l'ensemble de son œuvre : il y a tout d'abord un héros narrateur, qui va témoigner de l'aventure qui s'est produite, aventure décrite d'une façon rationnelle, mais qui débouche sur l'intrusion d'un irrationnel pourtant plausible, voire vraisemblable, en tout état de cause pouvant être soumis à une interprétation excluant tout surnaturel. Ce héros se meut dans un espace restreint, celui d'un château, fort peu décrit, mais que l'on peut aisément imaginer d'origine médiévale, ne serait-ce que par l'atmosphère topique qui y règne : un grand escalier, un pavage et surtout une grande salle où des cavaliers pénètrent à cheval. Le capitaine rencontre une jeune femme, qui devient rapidement, comme très souvent chez Owen, le centre focalisant l'attention du lecteur, et un vieillard, au sujet duquel le narrateur hésite sur la fonction de père ou de mari. Cet homme, qui a tiré un coup de feu d'une fenêtre, sans véritable raison, est présenté par la femme, la baronne Kurchkrinzi, comme ayant un peu perdu la tête. Comme le lecteur le pressent assez rapidement, la baronne va au soir conduire le capitaine, par des voies sombres et un peu angoissantes, vers une chambre où elle se donne à lui. Au matin, le capitaine découvre une momie à côté de lui. En s'échappant de la chambre, il découvre l'état de délabrement du château et aperçoit le vieillard qui pointe une arme vers lui. Il repart avec ses troupes.

Voici donc une nouvelle très classique, au schéma narratif plutôt simple et assez convenu, susceptible au demeurant d'être apprécié de l'amateur de fantastique qui retrouvera maint repère qui lui sont familiers, ce qui fait aussi le charme de tout texte s'inscrivant dans un fort contexte générique, ce que les auteurs médiévaux avaient largement cultivé, soit dit en passant, dans une culture et des mentalités où l'originalité est d'abord perçue comme un défaut.

Toutefois, Owen autorise également, comme il fait souvent, dans différentes directions, une lecture moins immédiate. Le titre de la nouvelle, « La nuit au château » 
suggère déjà un certain cadre et même renvoie, en forme d'interrogation, à diverses possibilités de cadrage : atmosphère médiévale de la halte dans un «ostel» du chevalier errant poursuivant sa quête ; nuit inquiétante en une bâtisse de type gothic novel, pourquoi pas non plus atmosphère sadienne ou encore occulte et mystérieuse à la façon Lovelace ou Péladan. L'entrée en matière nous présente un environnement dévasté : «Les carrés de buis saccagés, les pelouses piétinées, les meubles de jardin culbutés, un grand parasol blanc et noir éventré... ${ }^{14}$. Puis apparaissent les personnages de la jeune et belle femme, et du vieillard fortement handicapé. Le héros est ensuite conduit dans un parcours qu'il subit sans comprendre vraiment les enjeux et sans avoir l'idée de demander quoi que ce soit sur sa curieuse aventure. Enfin, le réveil révèle un château dévasté, vide. Le lecteur au fait du Conte du graal de Chrétien de Troyes ne sera pas, non sans délectation repérer les éléments qu'Owen lui offre en signe de reconnaissance, même réinterprétés et réordonnés. Le début de l'histoire, dans une chronologie inverse du roman de Chrétien, invite à penser à la « terre gaste » qui entoure le château du roi pêcheur. Le vieillard s'apparente au roi pêcheur, peut-être plus dans l'esprit de Wolfram von Eschenbach que de Chrétien. Enfin, le parcours du héros dans le château est une évocation, au trait léger, de l'aventure de Perceval au château du roi pêcheur où le jeune chevalier n'a pas osé poser de question et se retrouve ainsi au matin dans un univers vide et dévasté.

Cette nouvelle est précédée, dans l'édition des œuvres complètes d'Owen, par une autre dont la teneur médiévale est annoncée clairement dès le titre « La belle vaincue et le troubadour », qui appartient au groupe La Truie, paru en 1972. Dans ce texte, nous retrouvons, sous un autre angle, la façon de procéder d'Owen. Le début de la nouvelle est une parodie/clin d'œil de la célèbre Natureingang pratiquée par les troubadours :

Il faisait beau. Il faisait chaud. Il y avait des frous-frous de libellules dans les roseaux. Sur les prés planait le concert métallique des sauterelles et des grillons qui est comme le bruit de la chaleur. Au bord de la rivière, des aulnes laissaient pendre leurs branches basses pour les tremper doucement dans l'eau de temps en temps au gré de la toute petite brise. Aux endroits calmes, où le courant flâne un peu et se repose entre la berge et les massifs de joncs, des moustiques à détentes brusques et saccadées jouaient silencieusement en laissant à la surface de petites traces vite effacées.

En tendant l'oreille, on aurait entendu le petit ruisseau sautillant, tout froid de la fraîcheur des bois, faire rire et chatouiller les pierres de son lit. C'était lointain, cristallin, imperceptible...

Le ciel était tout bleu. Dans l'herbe haute, jouaient des papillons noirs et roux ${ }^{15}$.

Voici donc un temps de reverdie, mais marqué du sceau d'un humour assez caustique. S'il fait beau, si le ciel est bleu comme dans l'évocation de certaines amours

\footnotetext{
${ }^{14}$ Euvres complètes, t. 3, $1998: 167$.

${ }^{15}$ Euvres complètes, t. 3, $1998: 159$.
} 
troubadouresques, les oiseaux traditionnels de printemps, topiques chez les poètes courtois, sont ici remplacés par des grillons, des sauterelles, des papillons et même des moustiques !

L'histoire, une fois encore, a une trame assez simple : dans une barque, chante un troubadour pour une femme allongée, complètement nue, nommée « la belle vaincue ». Un homme, caché dans les buissons, donné de prime abord comme un chasseur - mais avec un visage «barré d'une épaisse moustache noire de mari trompé $»^{16}$, les observe avec son arme, met en joue, hésite, renonce, puis finit pas tirer sur le poète, dont la guitare explose. Ceci provoque le désespoir du troubadour qui se jette à l'eau et se noie sous les yeux totalement indifférents de la belle (« ... elle n'eut pas un regard pour l'eau subitement tragique où venait de disparaître le pauvre garçon $\left.»^{17}\right)$. Ayant regagné la rive, puis s'étant rhabillé, elle rejoint son chasseur de mari. Ce dernier lui fait la remarque qu'elle rentre tard, ce à quoi elle répond qu'elle revient de chez le dentiste. La conclusion est lapidaire : «- Ah ! Il aimait mieux ça... » mais c'est elle qui, comme souvent dans la nouvelle, donne tout le sens et le sel du texte.

Voici donc une nouvelle tragi-comique, avec un humour teinté de misogynie et d'un certain désabusement sur le mariage. On pourra remarquer que dans sa tonalité comme dans sa conclusion, la dénomination de 'troubadour' pour le chanteur est inutile. On peut très bien lire et goûter la nouvelle en remplaçant 'troubadour' par 'amant' ou 'chanteur' : une jeune et jolie femme adultère se promène en barque avec son amant ; elle est nue et le qualificatif de " vaincue » évoque qu'elle est alanguie après avoir fait l'amour. Son mari, jaloux et quelque peu ridicule (« coiffé d'un chapeau melon noir, risible et drôlement juché sur le haut de son crâne »), sans doute bien plus âgé qu'elle, surprend les amants et tire sur l'homme. La femme est en fait une libertine sans cœur et le mari accepte pour la garder, en dépit de sa jalousie, la comédie qu'elle lui joue.

Il y a cependant le terme troubadour qui, comme nous l'avons vu, peut imposer une lecture dès le début du texte qui, tout du long, va jouer en décalage avec les codes de la lyrique troubadouresque. La femme en effet est belle, supérieure, dominante, conformément à l'image de la domna dessinée par les troubadours. Les amants ont fait l'amour et Owen sait très bien que l'amour courtois, contrairement à quelques contresens répandus, n'a rien de platonique. La belle est mariée à un mari jaloux qui ne la satisfait pas et l'on retrouve le thème de la « maumariée », cher aux troubadours et trouvères du Moyen Âge. La mort du poète obéit aux mêmes règles ; la lyrique troubadouresque est en effet un jeu de miroir, miroir dans lequel le troubadour se reflète pour s'accomplir dans le joy, à savoir dans un amour souvent abstrait et surtout dans son art de composer, son art de trobar. Le mari détruit donc

\footnotetext{
${ }^{16}$ Euvres complètes, t. 3, $1998: 160$.

${ }^{17}$ Euvres complètes, t. 3, $1998: 162$.
} 
ici, non directement l'amant, mais son instrument qui fonctionne de façon métonymique comme le contenant de tout l'art d'aimer et de chanter. Le troubadour ne peut donc effectivement survivre à cette perte qui annihile sa propre essence.

Si Owen n'est pas à proprement parler un auteur 'médiévalisant', la mémoire du Moyen Âge agit cependant sur certaines de ses nouvelles et, le plus souvent par un effet de dramatisation. C'est dans un Moyen Âge angoissant, qui joue avec la mort, avec les ténèbres, avec l'incertitude que puise Owen: un Moyen Âge qui nous rappelle celui qu'évoquait Franz Hellens dans son premier roman En ville morte, où apparaît Gand en filigrane, «ville morte aux néfastes ruelles terrassées par l'ombre tyrannique du monstre médiéval ».

L'inscription de cette mémoire ne se fait pas, sauf dans le cas du professeur Sardon, de manière explicite et directe, sur le mode de la citation ou même de la récriture - il n'y a rien d'historique ici - mais d'une façon diffuse, souvent décalée. Le récit est infiltré de manière capillaire par un ensemble de références, qui ne sont audibles que d'un certain public. Owen joue ainsi avec sa propre culture, souvent avec humour et, par là, il offre une possibilité de lecture à décryptage qui transforme aussi la nouvelle en jeu de piste. Thomas Owen offre donc, même si c'est de façon anecdotique au regard de la masse considérable de ses nouvelles, un excellent exemple de la façon, que je considère comme très subtile, dont un écrivain contemporain peut se réapproprier la culture et la littérature du passé. La littérature médiévale étant pour une très large part une littérature à codes, ce qui en fait son intérêt, mais aussi sa difficulté d'approche pour le moderne, se prête donc particulièrement bien à ce type de réexploitation dont Owen a su tirer un excellent parti pour le plaisir du lecteur averti. 


\section{BIBLIOGRAPHIE}

Baudry, R. (1996). «Le Lancelot-Guenièvre de Jean Cocteau ou les Avatars du Mythe Amours, Sosies et Substitutions », CRMH, 37-49.

Chagas, O. E. (2008). «A Descoberta da Nova Retórica: Jean Paulhan e Brunetto Latini. La Découverte de la Nouvelle Rhétorique: Jean Paulhan et Brunetto Latini », Ideação, (Núcleo Interdisciplinar de Estudos e Pesquisas em Filosofia), 18/12, 17-32.

Dubost, F. (1991). Aspects fantastiques de la littérature narrative médiévale (XII ${ }^{e}-X I I I^{e}$ s.). L'autre, l'ailleurs, l'autrefois, Paris : Champion, 2 vol.

Lope, H. J., Neuschäfer, A. (ed.) (1998). René Kalisky (1936-1981) et la hantise de l'Histoire, éd., Frankfurt/Main - Berlin - New York - Paris - Wien : Peter Lang.

Marot, P. (1995). "Le Roi pêcheur : Le Graal ou l'envers de la représentation », Cahiers de l'Association internationale des études françaises, 47, 115-134.

Owen, Th. (1998). Euvres complètes, 4 vol., Bruxelles : Lefrancq.

Pomel, F. (2005). «Michel Rio et le modèle textuel médiéval: filiation et paternité littéraires », in Images du Moyen Âge, Presses Universitaires de Rennes.

- (2010). «Michel Rio : les réécritures arthuriennes », Cahiers de recherches médiévales et humanistes, 19, 145-149.

Ribémont, B. (2006). «Michel Rio : une réécriture de la légende arthurienne ou la mystique de la loi », in Mythosaktualisierungen. Tradierungs-und Generierungspotentiale einer alten Errinerugsform, Hrgs S. Wodianka, D. Rieger, Berlin - New York : De Gruyter, 193-209.

Ruelens, Ch. (1872). «La Bibliothèque bleue en Belgique », Le Bibliophile belge, 7, 59-69, 200-204.

Schneider, M. (1985). « Cocteau et le Moyen Âge », Cahiers Jean Cocteau, 10, 259-265.

Silvestri, A. (2003). «Charles le Téméraire ou l'autopsie d'un prince de René Kalisky : un défi contre la 'désexistence' ", in Les écrivains francophones interprètes de l'Histoire : entre filiation et dissidence. Actes du colloque de Cerisy-la-Salle, du 2 au 9 septembre 2003, Bruxelles : Peter Lang, 435-456. 
